\title{
Lichen simplex chronicus on the scalp: exuberant clinical, dermoscopic, and histopathological findings*
}

\author{
Bianca Pinheiro Bousquet Muylaert ${ }^{1}$ \\ Alexandre Ozores Michalany²
}

\author{
Mayara Trindade Borges ${ }^{1}$ \\ Camila Raposo Cabral Scuotto ${ }^{3}$
}

DOI: http:/ / dx.doi.org/10.1590/abd1806-4841.20186493

\begin{abstract}
Lichen simplex chronicus is a skin disease that mostly affects female patients, with a peak incidence between ages 35 and 50 years. On the scalp, it appears as a single or multiple oval lesions, showing scaling and hair shaft loss or breakage. An important dermoscopic feature of the disease are the "broom fibers." Histopathology reveals the "hamburger" and the "gear wheel" signs. The aim of this report is to demonstrate a case of lichen simplex chronicus on the scalp with typical and exuberant clinical, dermoscopic, and histopathological findings.
\end{abstract}

Keywords: Dermoscopy; Lichen; Scalp; Scalp dermatoses

\section{INTRODUCTION}

Lichen simplex chronicus is a cutaneous disorder characterized by lichenification of the skin as a result of intense excoriation secondary to excessive primary pruritus, becoming a self-perpetuating mechanism. ${ }^{1,2}$ It mostly affects female patients, with a peak incidence between ages 35 and 50 years. On the skin, it presents itself as a thickened and hyperpigmented lichenoid plaque. It mainly affects the cervical region, ankles, scalp, vulva, scrotum, and extensor region of the upper limbs. ${ }^{1}$ On the scalp, it manifests itself as a single or multiple oval lesions, with scaling, causing hair loss or breakage. ${ }^{3}$

Dermoscopy can show erythema and perifollicular scaling associated with hair shaft breakage. ${ }^{3}$ Histopathological examination of the scalp lesion reveals a preservation of the follicle architecture, with normal number of terminal follicles, but with a decrease in size and number of sebaceous glands. Characteristic features of dermatosis are present, and are reported below. ${ }^{1,3}$

\section{CASE REPORT}

A 75-year-old female patient, retired, born in the state of Minas Gerais, Brazil, but coming from the State of São Paulo, presented with a 10-year history of pruritus on the scalp that started to worsen two years before. She reported using antihypertensive and timolol eye drops and denied depression, anxiety, or other psychoses.

On physical examination, we observed two well-delimited hair rarefaction areas on the scalp, "broom fibers" displaying a tonsure pattern, polithychia, and moderate perifollicular desquamation in the left parietal-occipital and parietal regions (Figure 1).

Dermoscopy revealed erythema and desquamation, mainly perifollicular, as well as tonsure hair pattern in the proximal or distal parts of the shafts in both plaques (Figure 2). Anatomopathological examination of the scalp lesion revealed 28 hair follicles, 6 vellus hairs, and 22 terminal and intermediate hairs, as well as follicles with 2 or more hair shafts, preserved follicular epithelium, and absence of inflammatory infiltrate. The examination also revealed, in the infundibular ostium, hyperkeratosis with hairs split into two segments by a layer of red blood cells, as well as jagged acanthotic projections emerging from the outer root sheath (Figures 3 to 6).

After the diagnosis of lichen simplex chronicus, we opted for the use of occlusive topical clobetasol. After three months, we observed a significant symptom improvement with relief of pruritus and consequent cessation of repetitive trauma.

\footnotetext{
Received on 27.09.2016.

Approved by the Advisory Board and accepted for publication on 14.03.2017.
$*$ Work performed at the Dermatology Department of the Universidade de Santo Amaro (UNISA) - Santo Amaro (SP), Brazil.

Financial support: None.

Conflict of interest: None.

Dermatology Department at Universidade de Santo Amaro (UNISA) - Santo Amaro (SP), Brazil.

Dermatopathology Department at Universidade de Santo Amaro (UNISA) - Santo Amaro (SP), Brazil.

Thychology Outpatient Division at Universidade de Santo Amaro (UNISA) - Santo Amaro (SP), Brazil.
}

\section{MAILING ADDRESS:}

Bianca Pinheiro Bousquet Muylaert

E-mail: biamuylaert@hotmail.com 


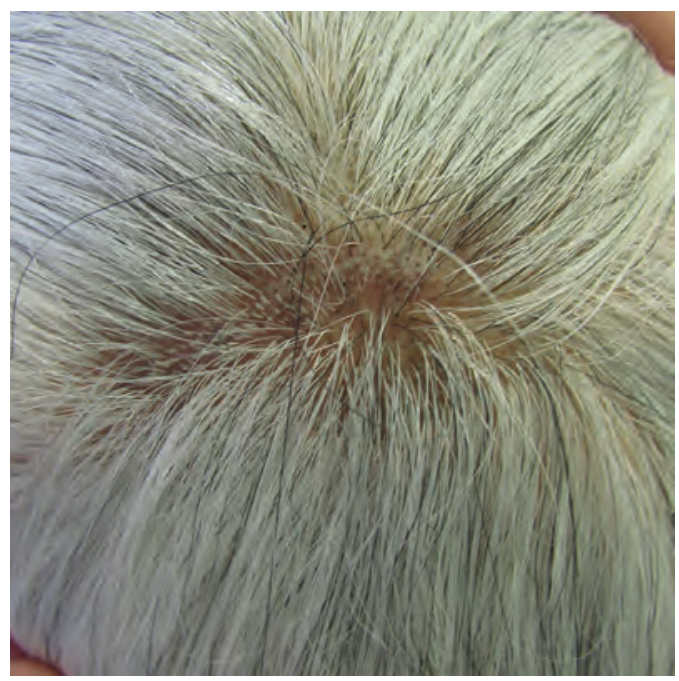

FIGURE 1: Well-delimited hair rarefaction area on the scalp, "broom fibers" displaying a tonsure pattern, politrychia, and moderate perifollicular scaling

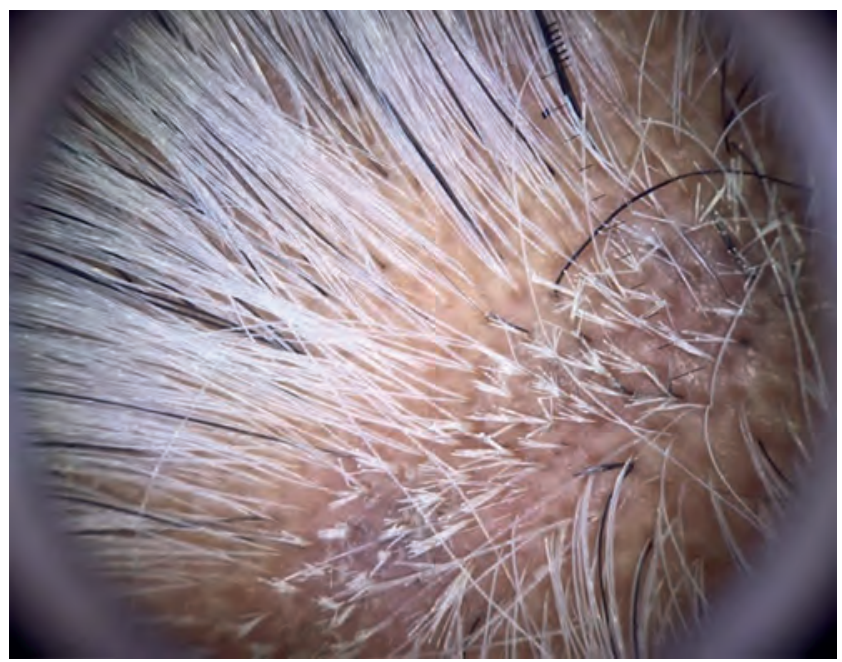

FiguRE 2: Dermoscopy: erythema, perifollicular desquamation, and tonsure pattern

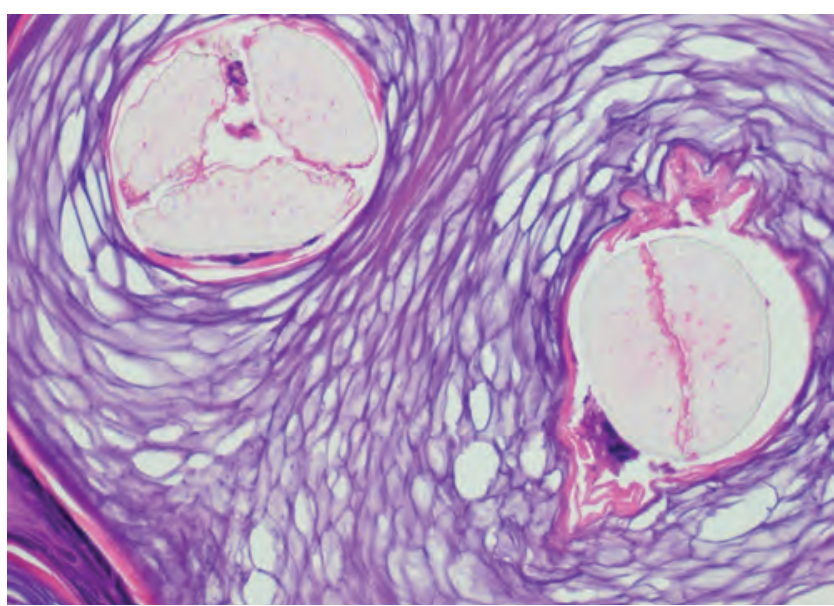

FIGURE 3: Hamburger sign: hyperkeratosis with hairs split into two segments by a layer of red blood cells (Hematoxylin \& eosin, X40)

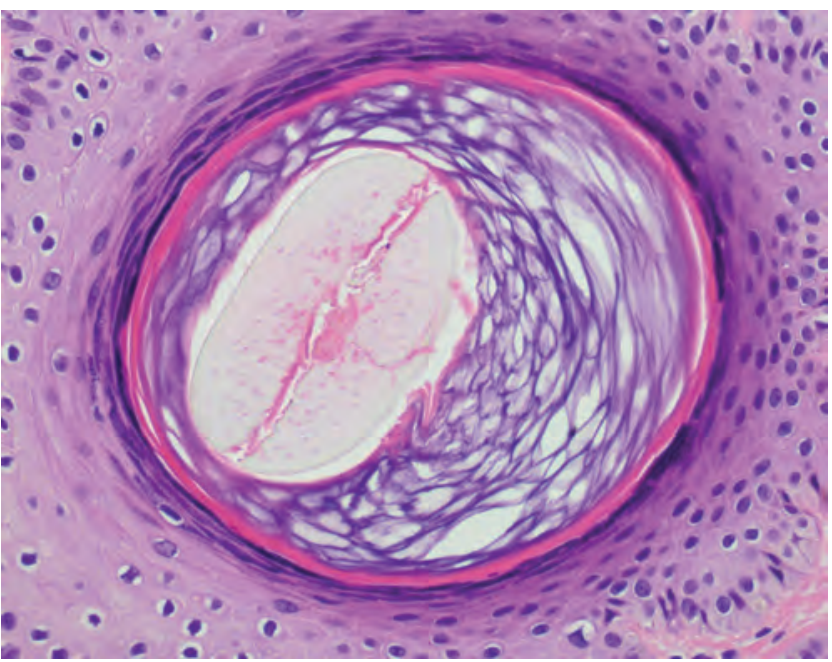

Figure 4: Hamburger sign at a higher magnification (Hematoxylin \& eosin, $\mathrm{X} 400)$

at the distal end of the hair. Such an abnormality is described as "broom fibers." 3 The present report revealed broom fibers as well as erythema and perifollicular desquamation. Usual histopathological features of lichen simplex chronicus include epidermal hyperplasia, orthokeratosis, and hypergranulosus with regular lengthening of the papillary ridges. A perivascular infiltration of lymphocytes and eventually macrophages is also commonly observed. ${ }^{1}$ Histopathological features of the scalp lesion include preservation of follicle architecture, with a normal number of terminal follicles, but with a decrease in size and number of sebaceous glands. At the infundibulum level, the outer root sheath forms acanthotic jagged projections, termed "gear wheels." The infundibular ostium shows hyperkeratosis with hair shaft split into two segments by a layer of erythrocytes, called the "hamburger sign". ${ }^{3}$ In the present case, as described 


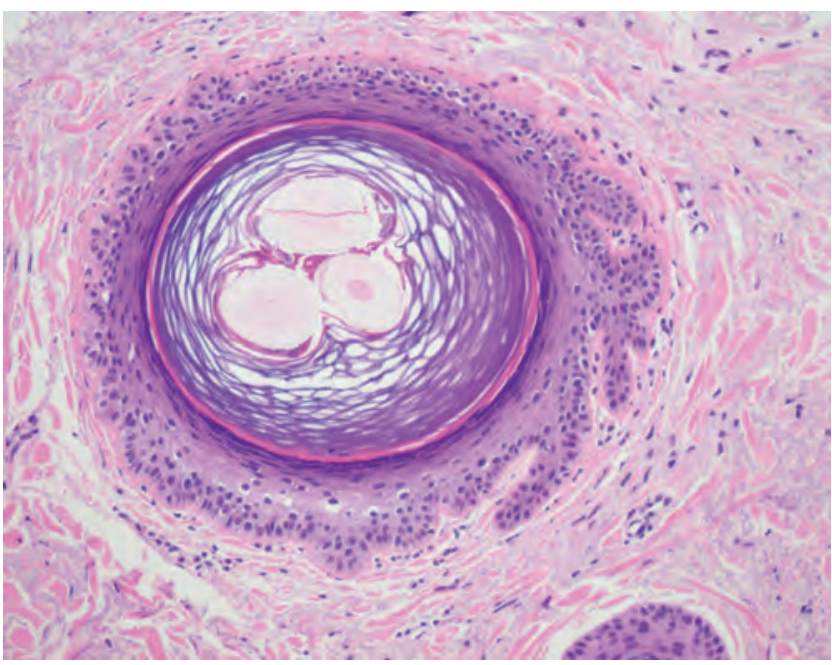

FigURE 5: Gear wheel sign: outer root sheath forms acanthotic jagged projections (Hematoxylin \& eosin, X40)

in the literature, we observed no change in the number of follicles or inflammatory infiltrate, with the presence of the characteristic signs described here. We also observed follicles with two or more hair shafts, which clinically manifested themselves as politrychia.

Trichotillomania may be a differential diagnosis since the "hamburger sign" in histopathology and the "broom sign" in dermoscopy are common features to both diseases. However, this disorder can be distinguished from lichen simplex chronicus for its broken hairs with different shaft lengths - termed sparse black and yellow dots - and rarely causes erythema and pruritus on the scalp. ${ }^{3,6}$

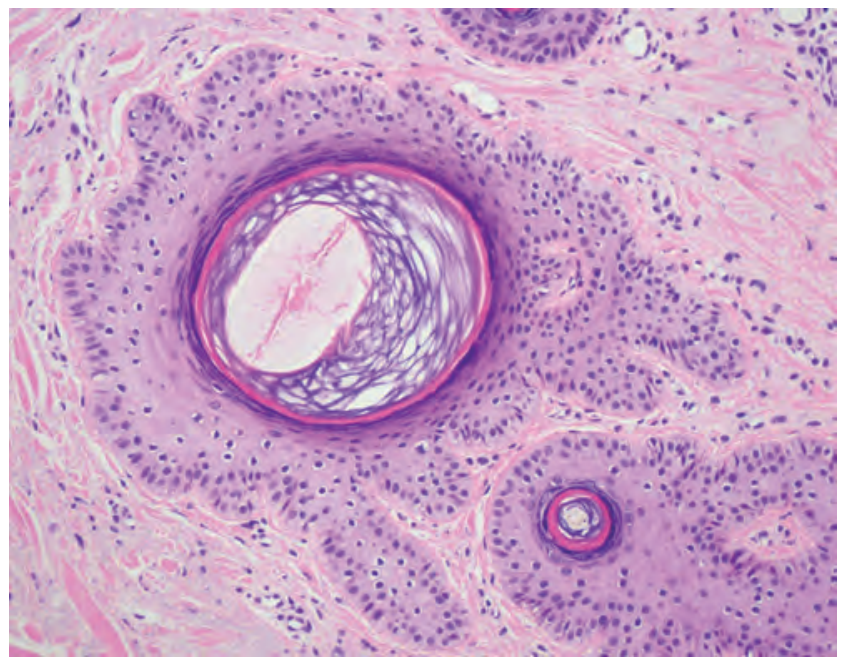

Figure 6: Hamburger sign and gear wheel sign (Hematoxylin \& eosin, $\mathrm{X} 40$ )

The goal of treatment of lichen simplex chronicus is to interrupt the process that causes pruritus and excoriation. High-potency topical or intralesional corticosteroids usually present satisfactory responses. Capsaicin topical and tacrolimus are also used. Psychotherapy is recommended as an adjuvant treatment. ${ }^{1,2}$ Our patient showed significant symptom improvement after three months of use of occlusive topical clobetasol.

The purpose of this report is to demonstrate a case of lichen simplex chronicus on the scalp with typical and exuberant clinical, dermoscopic, and histopathological findings.

\section{REFERENCES}

1. Ambika H, Vinod CS, Sushmita J. A Case of Neurodermatitis Circumscipta of Scalp Presenting as Patchy Alopecia. Int J Trichology. 2013;5:94-6.

2. Lotti T, Buggiani G, Prignano F. Prurigo nodularis and lichen simplex cronicus. Dermatol Ther. 2008;21:42-6.

3. Quaresma MV, Mariño Alvarez AM, Miteva M. Dermatoscopic-pathologic correlation of lichen simplex chronicus on the scalp: 'broom fibres, gear wheels and hamburgers'. J Eur Acad Dermatol Venereol. 2016;30:343-5.

4. Stillians AW. Lichen simplex of scalp. Arch Derm Syphilol. 1926;13:819-21.

5. Miteva M, Tosti A. Dermatoscopy of hair shaft disorders. J Am Acad Dermatol. 2013;68:473-81.

6. Royer MC, Sperling LC. Splitting hairs: the "hamburger sign" in trichotillomania. J Cutan Pathol. 2006;33:63-4.

How to cite this article: Muylaert BPB, Borges MT, Michalany AO, Scuotto CRC. Lichen simplex chronicus on the scalp: exuberant clinical, dermoscopic, and histopathological findings. An Bras Dermatol. 2018;93(1):108-10. 